\title{
Experimental insights into the coupling of methane combustion and steam reforming in a catalytic plate reactor in transient mode
}

M. Arsalan Ashraf ${ }^{\mathrm{a}, \mathrm{d}, *}$, Stefano Tacchino ${ }^{\mathrm{a}}$, Nageswara Rao Peela ${ }^{\mathrm{b}, \mathrm{c}}$, Giuliana Ercolino ${ }^{\mathrm{a}}$, Kirandeep K. Gill ${ }^{\mathrm{d}}$, Dionisios G. Vlachos ${ }^{\mathrm{b}}$, Stefania Specchia ${ }^{\mathrm{a}, *}$

${ }^{\text {a }}$ Department of Applied Science and Technology, Politecnico di Torino, Torino 10129, Italy

${ }^{b}$ Department of Chemical and Biomolecular Engineering, University of Delaware-Newark, DE 19716-3110, USA

${ }^{c}$ Department of Chemical Engineering, Indian Institute of Technology, Guwahati 781039, India

${ }^{\mathrm{d}}$ Department of Chemical Engineering, University of Bath, Bath, BA2 7AY, United Kingdom

* Corresponding authors. $\quad$ E-mail: muhammad.ashraf@polito.it (M.A. Ashraf) stefania.specchia@polito.it (S. Specchia)

\section{S. Supplementary information}

\section{S1. Additional details of calculation}

The molecular weight of gas mixture $\left(M_{m i x}\right)$ is calculated by [1]

$M_{\text {mix }}=\sum_{i=1}^{n} y_{i} M_{i}$

The density of gas mixture $\left(\rho_{\text {mix }}\right)$ is calculated as [1]

$\rho_{\text {mix }}=\frac{P M_{\text {mix }}}{R T}$

where $y_{i}$ is the mole fraction of the compound, $M_{i}$ is the molecular weight of the compound $(\mathrm{g} / \mathrm{mol}), \mathrm{P}$ is the pressure $(\mathrm{kPa}), T$ is the absolute temperature $(\mathrm{K}), R$ is the universal gas constant $(\mathrm{J} / \mathrm{mol} . \mathrm{K})$. Gas viscosity $(\mu)$ of a single component is calculated from a corresponding state method: for $\mathrm{CH}_{4}, \mathrm{CO}_{2}, \mathrm{CO}$ and $\mathrm{N}_{2}$. It is calculated by [2]

$\mu \delta=64.008 \times 10^{-5}\left(4.5 T_{r}-1.67\right)^{0.625}$

$\oint=0.176 T_{c}^{1 / 6} M^{-1 / 2} P_{c}^{-2 / 3}$

$T_{r}=\frac{T}{T_{c}}$ 
where $T_{c}$ is the critical temperature $(\mathrm{K}), P_{c}$ is the critical pressure $(\mathrm{kPa}), T_{r}$ is the reduced temperature and $P_{r}$ is the reduced pressure $(\mathrm{kPa})$. For $\mathrm{H}_{2}$, the viscosity $\left(\mu_{\mathrm{H}_{2}}\right)$ is calculated by

$\mu_{H_{2}}=326.55 \times 10^{-5}(0.1375 T-1.167)^{0.625}$

For $\mathrm{H}_{2} \mathrm{O}$, the viscosity $\left(\mu_{\mathrm{H}_{2} \mathrm{O}}\right)$ is calculated by

$\mu_{H_{2} \mathrm{O}}=1170.828 \times 10^{-5}(0.011662 \mathrm{~T}-0.551)$

The viscosity of gas mixture $\left(\mu_{m i x}\right)$ is calculated by [1]

$\mu_{\text {mix }}=\frac{\sum_{i=1}^{n} \mu_{i} y_{i} M_{i}^{1 / 2}}{\sum_{i=1}^{n} y_{i} M_{i}^{1 / 2}}$

The binary diffusion $\left(D_{A B}\right)$ of gas mixture species $\mathrm{i}$ and $\mathrm{j}$ is calculated by Fuller equation [3].

$$
D_{A B}=\frac{10^{-3} T^{1.75}\left(\frac{1}{M_{i}}+\frac{1}{M_{j}}\right)^{1 / 2}}{P_{T}\left(\sum v_{i}^{\frac{1}{3}}+\sum v_{j}^{\frac{1}{3}}\right)^{2}}
$$

The diffusivity of methane $\left(D_{i, m}\right)$ in gas phase is estimated by the following formula

$$
D_{i, m}=\frac{1-Y_{i}}{\sum_{j=1, j \neq i}^{N} \frac{Y_{i}}{D_{i, j}}}
$$

where $\mathrm{Yi}=$ mole fraction of methane, $v_{i}$ is the molar volume of compound. Knudsen diffusion $\left(D_{k}\right)$ is computed by [4]

$$
D_{k}=9.7 \times 10^{-5} r_{p} \sqrt{\frac{T}{M_{i}}}
$$

where $r_{p}$ is pore radius in Angstroms. The effective diffusivity of methane $\left(D_{i, e}\right)$ in washcoat is calculated using the following

$D_{i, e}=\frac{\varepsilon_{p}}{\tau}\left(\frac{1}{D_{i, m}}-\frac{1}{D_{k}}\right)^{-1}$

where $\varepsilon_{p}$ is the washcoat porosity with a typical value of 0.5 [5] is considered in this work and $\tau$ is the tortuosity factor taken as 8.1 for the washcoat as determined by Hayes et al., [6] study to evaluate the effective diffusivity of methane in nitrogen in the washcoat of ceramic monolith. The Sherwood number for gases is calculated as $[7,8]$ 
$S_{h}=\mathrm{Nu}_{\square}\left(1+0.095 R_{e} S_{c} \frac{d_{h}}{L_{c}}\right)^{0.45}$

where the value of $N u_{\square}$ for square channel is $2.976, d_{h}$ is hydraulic diameter $(\mathrm{m})$, and $L_{c}$ is the channel length $(\mathrm{m})$.

$S_{h}=\frac{k_{G} d_{h}}{D_{\mathrm{i}, \mathrm{m}}}$

The Schmidt number $\left(S_{c}\right)$ is calculated as

$S_{c}=\frac{\mu_{m i x}}{\rho_{\text {mix }} D_{i, m}}$

Reynold number $\left(R_{e}\right)$ is calculated as

$R_{e}=\frac{d_{h} \times u_{S T P} \times \rho_{\text {mix }}}{\mu_{\text {mix }}}$

Weight hourly space velocity (WHSV) is estimated as

$W H S V=\frac{Q}{m_{\text {cat }}}$

The residence time is calculated as:

$t_{c}=\frac{L_{c}}{u}$

where $\mathrm{Q}$ is the flow rate, $\mathrm{m}_{\text {cat }}$ is catalyst weight deposited, and $u$ is the feed velocity. The Carberry criteria [9] is used to evaluate the existence of external mass transport limitation for first order reaction here with respect to methane.

$$
\mathrm{Ca}=\frac{r_{\text {obs }} \rho_{\text {cat }}}{k_{G} a_{m} C_{C H_{4}}}<0.05
$$

where $K_{G}$ is the external mass transfer between the gas mixture and the washcoat and $a_{m}$ is specific geometric surface area of channel, $\mathrm{C}_{\mathrm{CH}_{4}}$ is methane concentration in feed, $r_{\text {obs }}$ is the observed rate of reaction with respect to methane. The Weisz-Prater criterion $(\Psi)[10]$ is used to estimate the existence of washcoat diffusion limitation.

$\Psi=\left(\frac{r_{o b s} \rho_{c a t} \delta_{c}^{2}}{D_{i, e} \times C_{C H_{4}}}\right)<1$

\section{References}


[1] A. Bahadori, Natural gas processing technology and engineering design, in, Gulf Professional Pub.,, Waltham, MA, 2014.

[2] S.S.E.H. Elnashaie, S.S. Elshishini, Modelling, simulation, and optimization of industrial fixed bed catalytic reactors, Gordon and Breach Science Publishers, Yverdon, Switzerland ; Langhorne, Pa., 1993. [3] E.N. Fuller, P.D. Schettle, J.C. Giddings, A New Method for Prediction of Binary Gas-Phase Diffusion Coeffecients, Ind Eng Chem, 58 (1966) 18-27.

[4] C.N. Satterfield, Mass transfer in heterogeneous catalysis, M.I.T. Press, Cambridge, Mass., 1969.

[5] C. Cao, N. Zhang, Y. Cheng, Numerical analysis on steam methane reforming in a plate microchannel reactor: Effect of washcoat properties, Int J Hydrogen Energ, 41 (2016) 18921-18941. [6] R.E. Hayes, S.T. Kolaczkowskib, P.K.C. Li, S. Awdry, Evaluating the effective diffusivity of methane in the washcoat of a honeycomb monolith, Appl Catal B-Environ, 25 (2000) 93-104.

[7] R.K. Shah, A.L. London Laminar flow forced convection heat transfer and flow friction in straight and curved ducts - a summary of analytical solutions, in, California, 1971.

[8] N. Kockmann, Transport phenomena in micro process engineering, Springer, Berlin ; New York, 2008.

[9] F.H.M. Dekker, A. Bliek, F. Kapteijn, J.A. Moulijn, Analysis of mass and heat transfer in transient experiments over heterogeneous catalysts, Chemical Engineering Science, 50 (1995) 3573-3580.

[10] D.E. Boldrini, J.F. Sanchez, G.M. Tonetto, D.E. Damiani, Monolithic Stirrer Reactor: Performance in the Partial Hydrogenation of Sunflower Oil, Ind Eng Chem Res, 51 (2012) 12222-12232. 
Table S1. Operating conditions of catalytic methane combustion for co-current flow mode.

\begin{tabular}{|c|c|c|c|c|c|c|}
\hline Energy Input & $\begin{array}{c}\text { Methane } \\
\text { Flow }\end{array}$ & $\begin{array}{c}\text { Air } \\
\text { Flow }\end{array}$ & WHSV & \multicolumn{2}{c|}{ Contact Time } & $\begin{array}{c}\text { Channel Inlet } \\
\text { Velocity }\end{array}$ \\
\hline Wth (J/sec) & $\mathrm{ml} / \mathrm{min}$ & $\mathrm{ml} / \mathrm{min}$ & $\mathrm{NL} / \mathrm{hr} / \mathrm{gcat}$ & $\mathrm{msec}$ & $\mu \mathrm{sec}$ & $\mathrm{m} / \mathrm{sec}$ \\
\hline 38 & 63.7 & 846.7 & 534.0 & 9.5 & 9466.2 & 5.3 \\
\hline 88 & 147.6 & 1960.8 & 1236.6 & 4.1 & 4087.7 & 12.2 \\
\hline 112 & 187.8 & 2495.6 & 1573.9 & 3.2 & 3211.7 & 15.6 \\
\hline 180 & 301.9 & 4010.8 & 2529.4 & 2.0 & 1998.4 & 25.0 \\
\hline 244 & 409.2 & 5436.8 & 3428.8 & 1.5 & 1474.2 & 33.9 \\
\hline 274 & 459.5 & 6105.3 & 3850.3 & 1.3 & 1312.8 & 38.1 \\
\hline 289 & 484.7 & 6439.5 & 4061.1 & 1.2 & 1244.7 & 40.2 \\
\hline 310 & 519.9 & 6907.4 & 4356.2 & 1.2 & 1160.4 & 43.1 \\
\hline 329 & 551.8 & 7330.8 & 4623.2 & 1.1 & 1093.4 & 45.7 \\
\hline 409 & 686.0 & 9113.4 & 5747.4 & 0.9 & 879.5 & 56.9 \\
\hline 438 & 734.6 & 9759.5 & 6154.9 & 0.8 & 821.3 & 60.9 \\
\hline
\end{tabular}

Table S2. Operating conditions of catalytic methane combustion for counter-current flow mode.

\begin{tabular}{|c|c|c|c|c|c|c|}
\hline Energy Input & $\begin{array}{c}\text { Methane } \\
\text { Flow }\end{array}$ & $\begin{array}{c}\text { Air } \\
\text { Flow }\end{array}$ & WHSV & \multicolumn{2}{|c|}{ Contact Time } & $\begin{array}{c}\text { Channel Inlet } \\
\text { Velocity }\end{array}$ \\
\hline Wth (J/sec) & ml/min & ml/min & NL/hr/gcat & msec & $\mu s e c$ & $\mathrm{~m} / \mathrm{sec}$ \\
\hline 59 & 99.0 & 1314.6 & 829.1 & 6.1 & 6096.9 & 8.2 \\
\hline 75 & 125.8 & 1671.2 & 1053.9 & 4.8 & 4796.2 & 10.4 \\
\hline 59 & 99.0 & 1314.6 & 829.1 & 6.1 & 6096.9 & 8.2 \\
\hline 49 & 82.2 & 1091.8 & 688.6 & 7.3 & 7341.1 & 6.8 \\
\hline 38 & 63.7 & 846.7 & 534.0 & 9.5 & 9466.2 & 5.3 \\
\hline 49 & 82.2 & 1091.8 & 688.6 & 7.3 & 7341.1 & 6.8 \\
\hline 59 & 99.0 & 1314.6 & 829.1 & 6.1 & 6096.9 & 8.2 \\
\hline 68 & 114.0 & 1515.2 & 955.6 & 5.3 & 5289.9 & 9.5 \\
\hline 75 & 125.8 & 1671.2 & 1053.9 & 4.8 & 4796.2 & 10.4 \\
\hline 99 & 166.0 & 2205.9 & 1391.2 & 3.6 & 3633.5 & 13.8 \\
\hline 110 & 184.5 & 2451.0 & 1545.8 & 3.3 & 3270.1 & 15.3 \\
\hline 121 & 202.9 & 2696.1 & 1700.3 & 3.0 & 2972.8 & 16.8 \\
\hline 131 & 219.7 & 2918.9 & 1840.9 & 2.7 & 2745.9 & 18.2 \\
\hline 142 & 238.2 & 3164.0 & 1995.4 & 2.5 & 2533.2 & 19.7 \\
\hline 110 & 184.5 & 2451.0 & 1545.8 & 3.3 & 3270.1 & 15.3 \\
\hline 75 & 125.8 & 1671.2 & 1053.9 & 4.8 & 4796.2 & 10.4 \\
\hline 99 & 166.0 & 2205.9 & 1391.2 & 3.6 & 3633.5 & 13.8 \\
\hline 110 & 184.5 & 2451.0 & 1545.8 & 3.3 & 3270.1 & 15.3 \\
\hline 121 & 202.9 & 2696.1 & 1700.3 & 3.0 & 2972.8 & 16.8 \\
\hline 131 & 219.7 & 2918.9 & 1840.9 & 2.7 & 2745.9 & 18.2 \\
\hline 142 & 238.2 & 3164.0 & 1995.4 & 2.5 & 2533.2 & 19.7 \\
\hline & & & & & & \\
\hline
\end{tabular}


Table S3. Average inlet velocity in the center of channels in CPR.

\begin{tabular}{|c|c|c|c|c|c|c|c|c|c|c|c|}
\hline $\begin{array}{c}\text { CPR Inlet } \\
\text { Flow }\end{array}$ & \multicolumn{10}{|c|}{ Average velocity in the center of channel } \\
\hline NL/min & C-1 & C-2 & C-3 & C-4 & C-5 & C-6 & C-7 & C-8 & C-9 & C-10 & Average \\
\hline 0.4 & 1.4 & 1.4 & 1.4 & 1.4 & 1.4 & 1.4 & 1.4 & 1.4 & 1.5 & 1.5 & 1.4 \\
\hline 1.4 & 5 & 5.1 & 5 & 5.1 & 5.1 & 5.1 & 5.2 & 5.2 & 5.2 & 5.2 & 5.1 \\
\hline 3.2 & 11.6 & 11.7 & 11.6 & 11.7 & 11.7 & 11.7 & 12 & 12 & 12.2 & 12.1 & 11.8 \\
\hline 4 & 14.7 & 14.8 & 14.8 & 14.9 & 14.9 & 14.8 & 15.2 & 15.3 & 15.5 & 15.4 & 15 \\
\hline 6.4 & 23.4 & 23.6 & 23.5 & 23.6 & 23.6 & 23.5 & 24.2 & 24.3 & 24.6 & 24.5 & 23.9 \\
\hline 8.7 & 31.5 & 31.7 & 31.7 & 31.9 & 31.8 & 31.7 & 32.7 & 32.8 & 33.3 & 33.1 & 32.2 \\
\hline 9.8 & 35.2 & 35.5 & 35.5 & 35.7 & 35.7 & 35.5 & 36.7 & 36.8 & 37.3 & 37.1 & 36.1 \\
\hline 10.3 & 37.1 & 37.4 & 37.4 & 37.6 & 37.6 & 37.4 & 38.6 & 38.7 & 39.3 & 39.1 & 38 \\
\hline 11.1 & 39.8 & 40.1 & 40.1 & 40.4 & 40.3 & 40.1 & 41.4 & 41.6 & 42.1 & 41.9 & 40.8 \\
\hline 11.8 & 42.2 & 42.6 & 42.5 & 42.8 & 42.7 & 42.6 & 44 & 44.1 & 44.7 & 44.5 & 43.3 \\
\hline 14.7 & 52.2 & 52.7 & 52.7 & 53 & 52.9 & 52.7 & 54.5 & 54.6 & 55.4 & 55.1 & 53.6 \\
\hline 15.7 & 55.8 & 56.3 & 56.3 & 56.7 & 56.6 & 56.3 & 58.3 & 58.4 & 59.2 & 59 & 57.3 \\
\hline
\end{tabular}




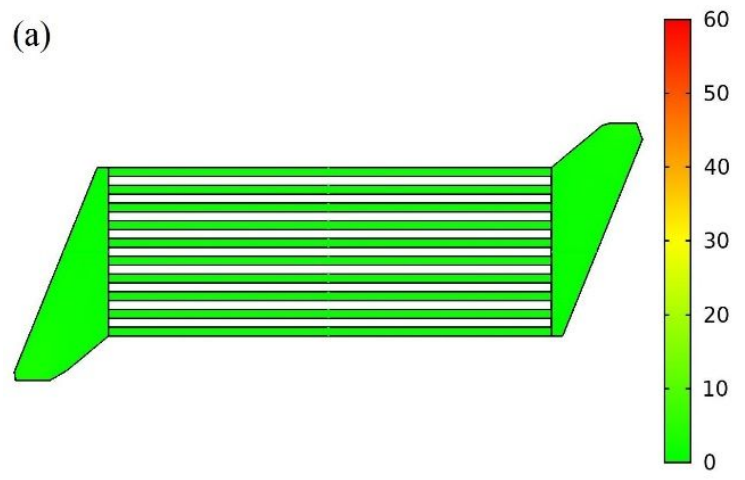

Channel inlet velocity $=1.4 \mathrm{~m} / \mathrm{s}$

(c)

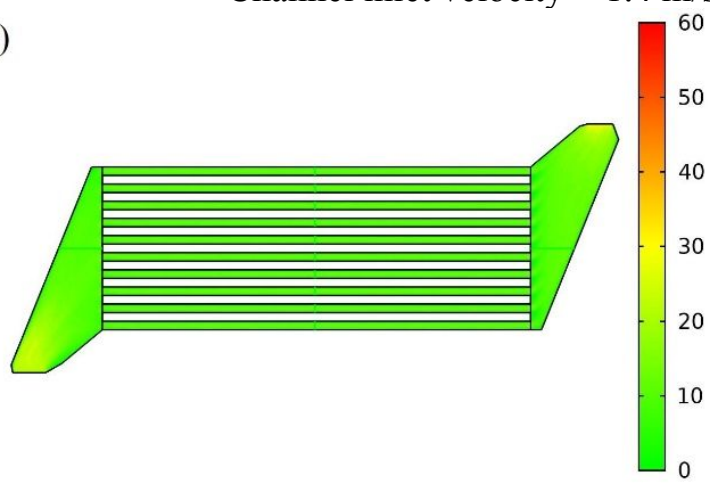

(e)

Channel inlet velocity $=11.8 \mathrm{~m} / \mathrm{s}$

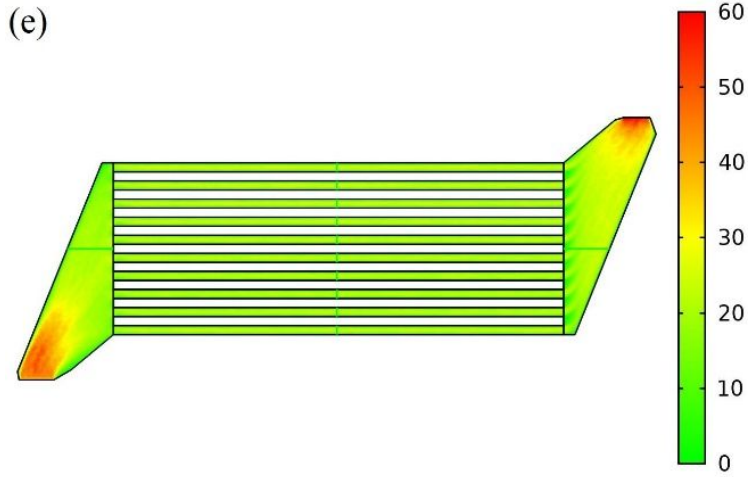

Channel inlet velocity $=23.9 \mathrm{~m} / \mathrm{s}$

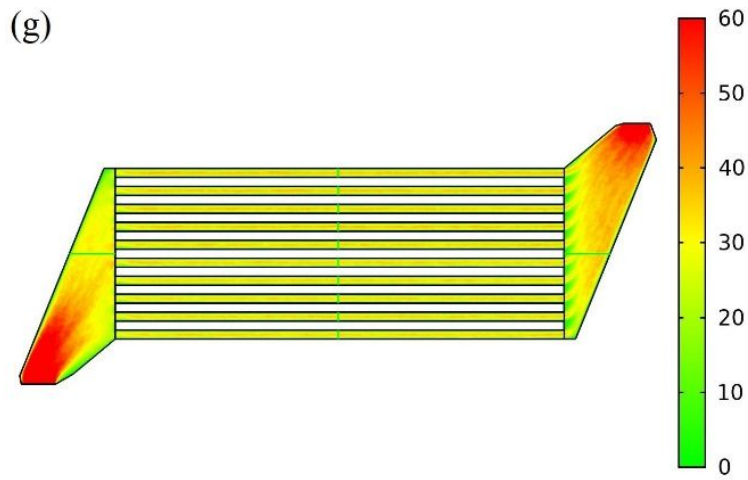

Channel inlet velocity $=36.1 \mathrm{~m} / \mathrm{s}$

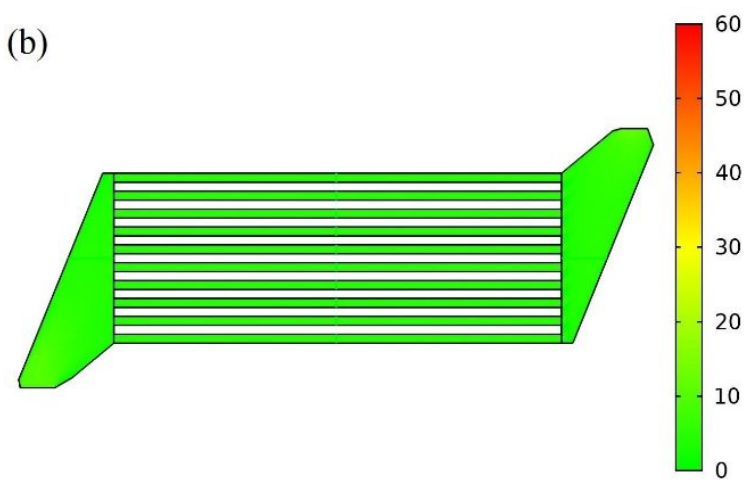

Channel inlet velocity $=5.1 \mathrm{~m} / \mathrm{s}$

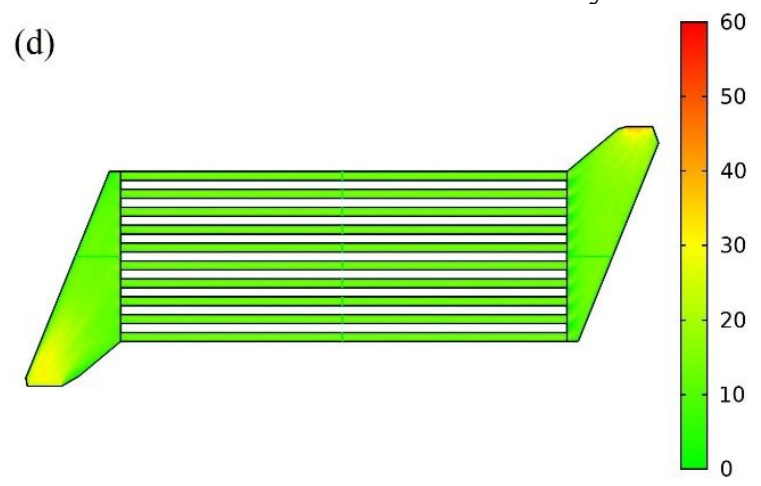

Channel inlet velocity $=15.0 \mathrm{~m} / \mathrm{s}$

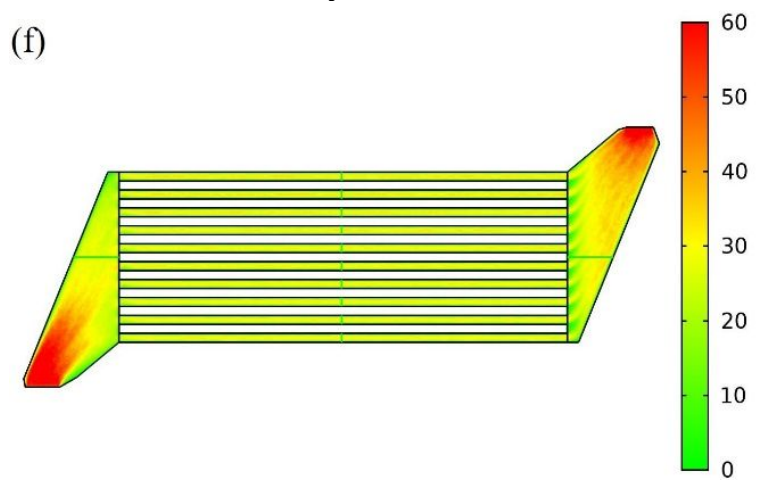

Channel inlet velocity $=32.2 \mathrm{~m} / \mathrm{s}$

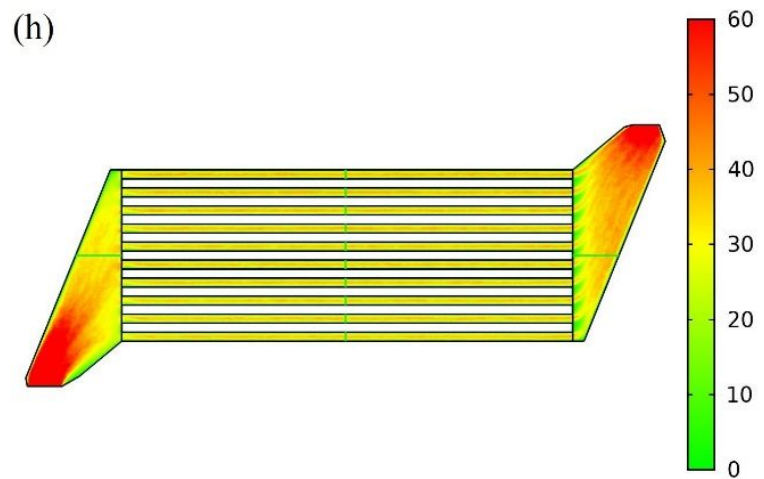

Channel inlet velocity $=38.0 \mathrm{~m} / \mathrm{s}$ 


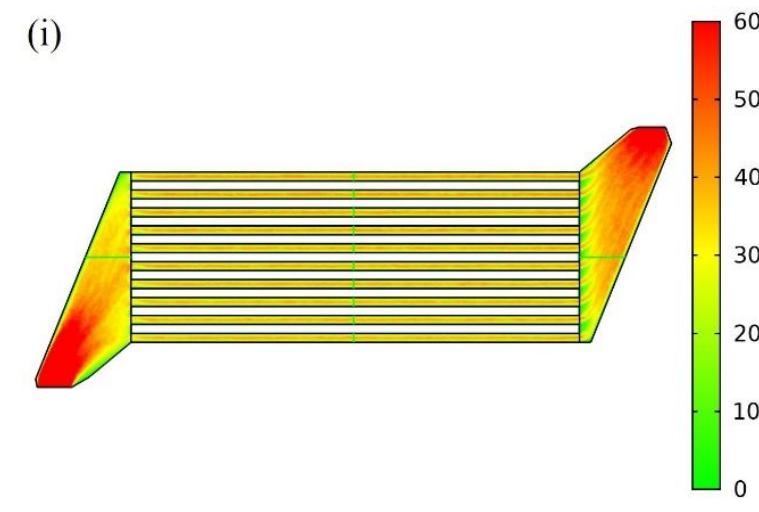

Channel inlet velocity $=40.8 \mathrm{~m} / \mathrm{s}$

(k)

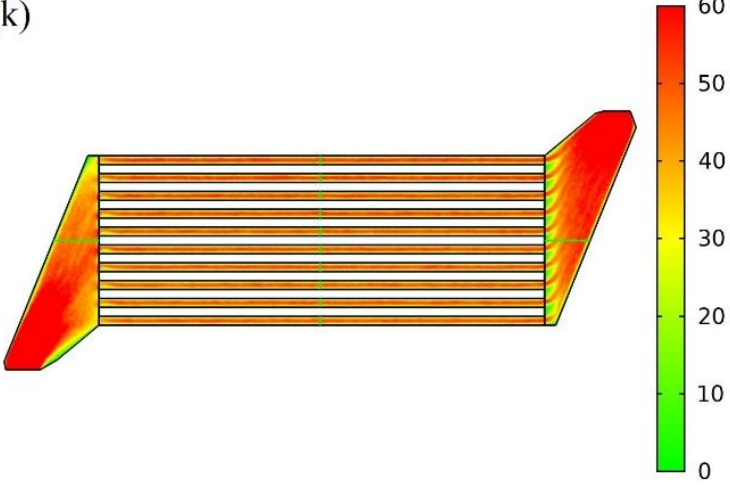

Channel inlet velocity $=53.6 \mathrm{~m} / \mathrm{s}$

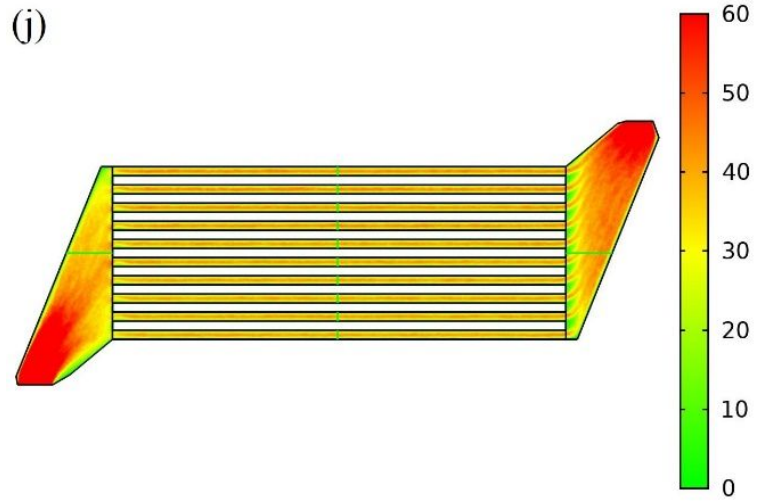

Channel inlet velocity $=43.3 \mathrm{~m} / \mathrm{s}$

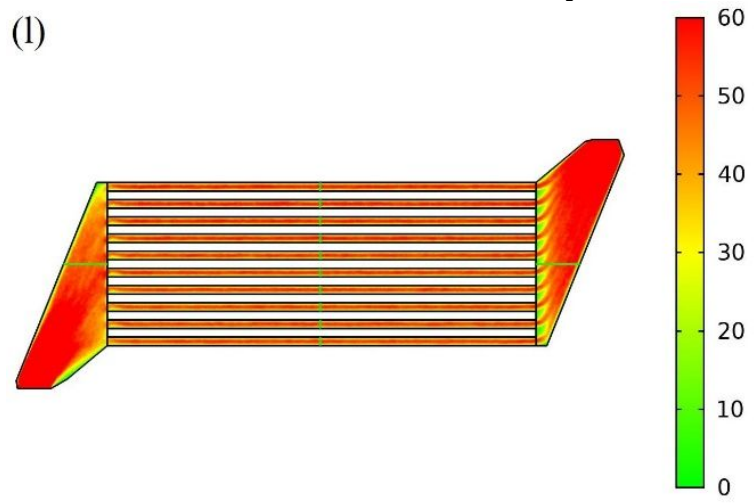

Channel inlet velocity $=57.3 \mathrm{~m} / \mathrm{s}$

Figure S1 $(\mathrm{a}-\mathrm{I})$. The velocity profile in microchannels of catalytic plate reactor at channel inlet velocity from 1.4 to $57.3 \mathrm{~m} / \mathrm{s}$.

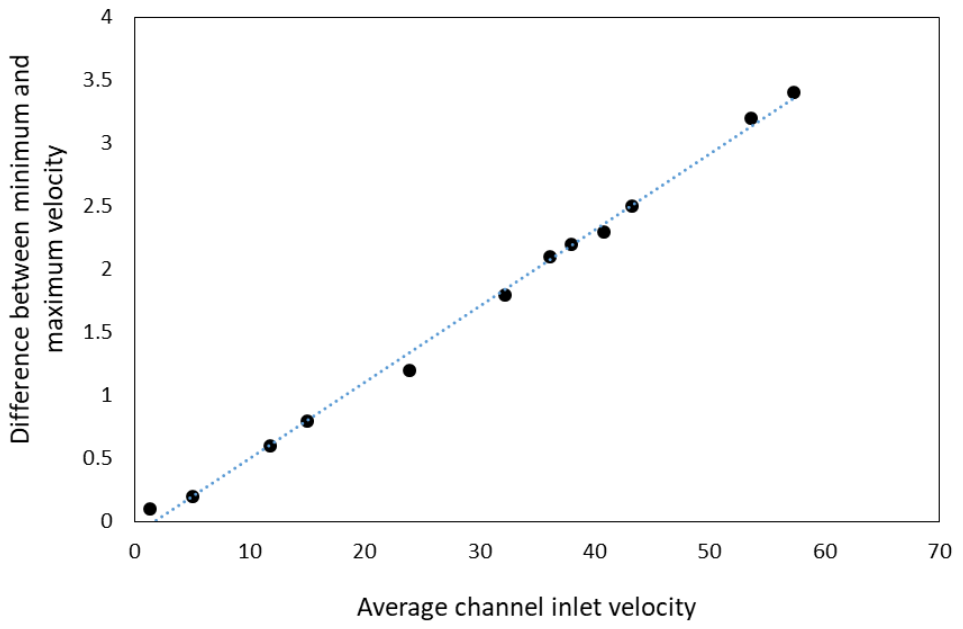

Figure S2: Difference between minimum and maximum velocities $(\mathrm{m} / \mathrm{s})$ versus average channel inlet velocity. 
(a)

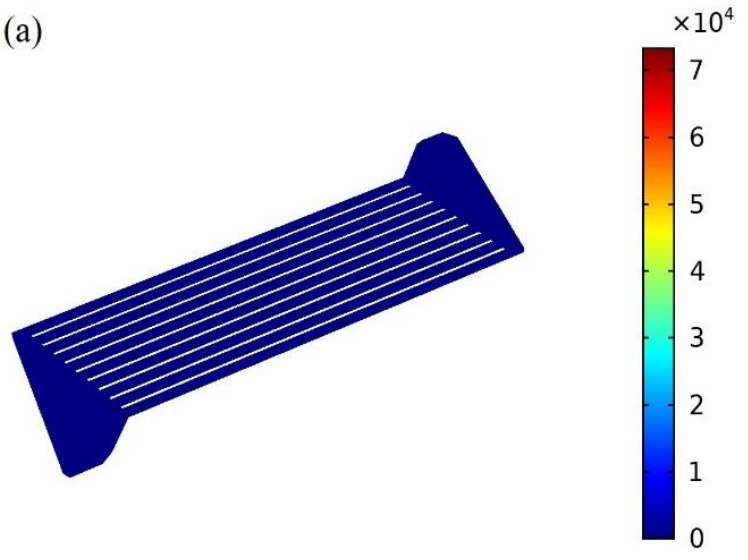

Channel inlet velocity $=1.4 \mathrm{~m} / \mathrm{s}$

(c)

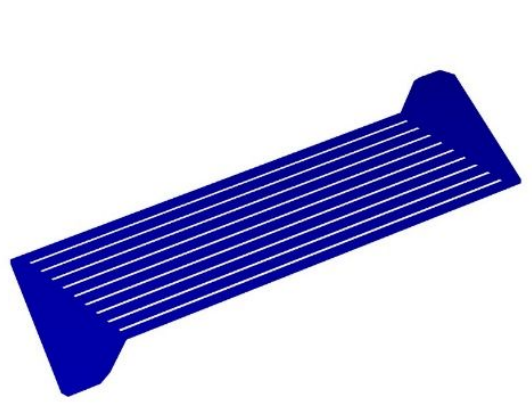

$\times 10^{4}$

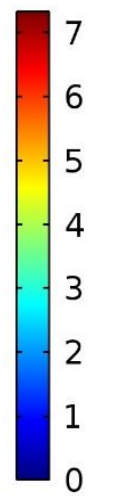

Channel inlet velocity $=11.8 \mathrm{~m} / \mathrm{s}$

(e)

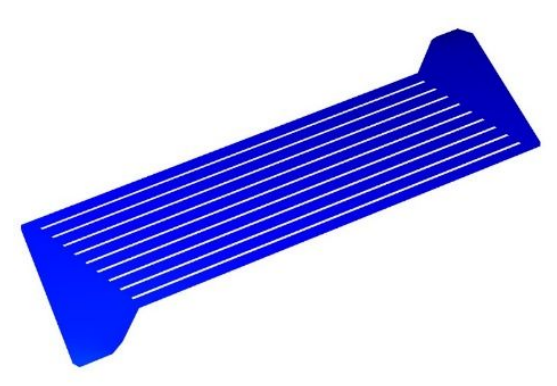

(b)

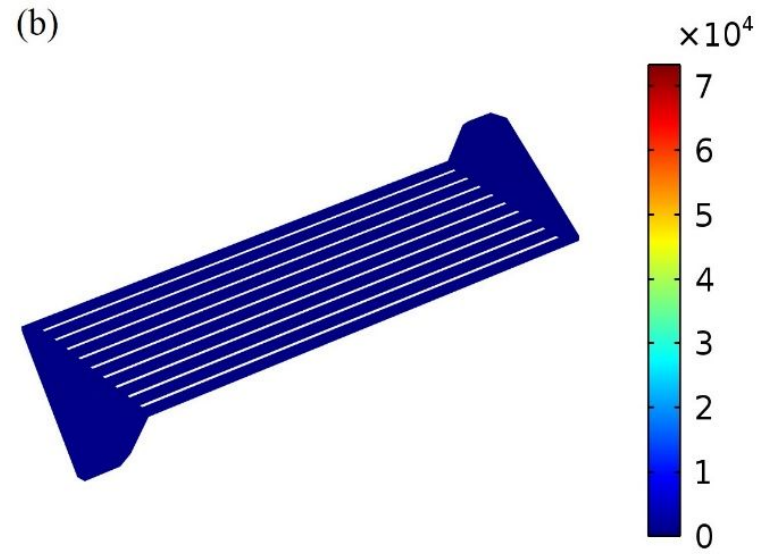

(d)

Channel inlet velocity $=5.1 \mathrm{~m} / \mathrm{s}$

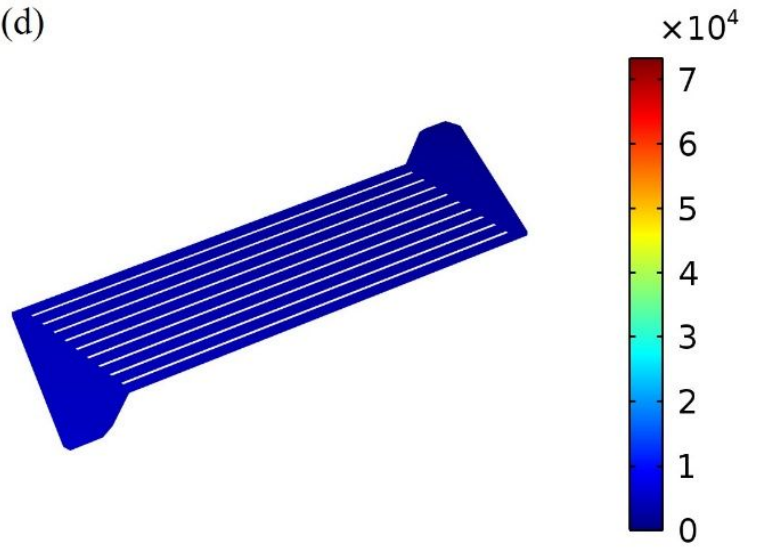

Channel inlet velocity $=15.0 \mathrm{~m} / \mathrm{s}$

(f)

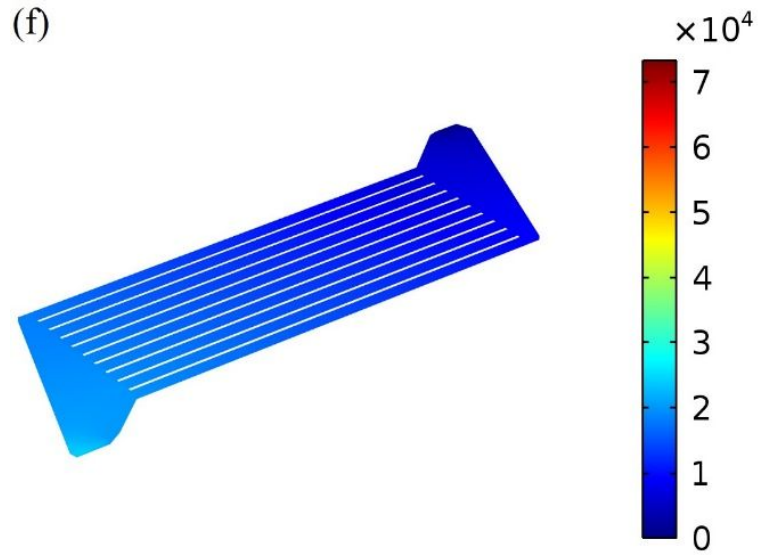

Channel inlet velocity $=32.2 \mathrm{~m} / \mathrm{s}$
4
Channel inlet velocity $=23.9 \mathrm{~m} / \mathrm{s}$

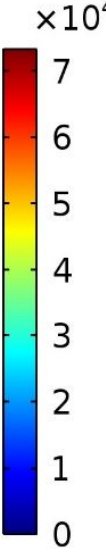
$\times 10^{4}$

$-\begin{aligned} & 7 \\ & 6 \\ & 5 \\ & 3 \\ & 2 \\ & 1 \\ & 0\end{aligned}$


(g)

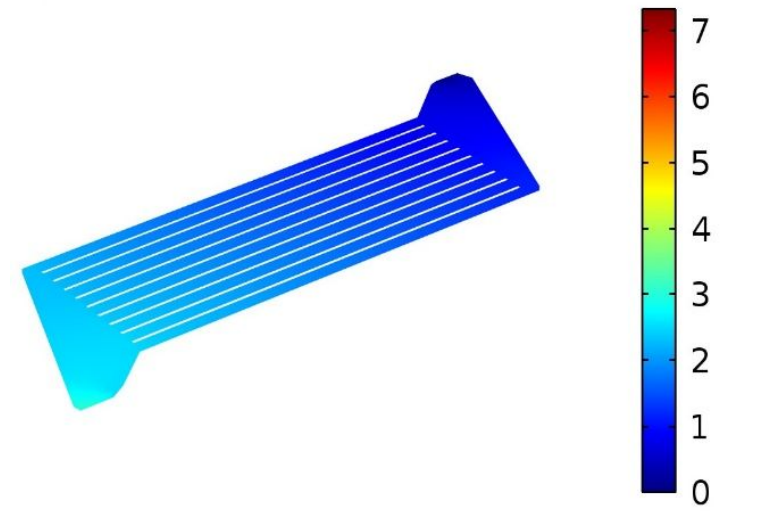

Channel inlet velocity $=36.1 \mathrm{~m} / \mathrm{s}$

(i)

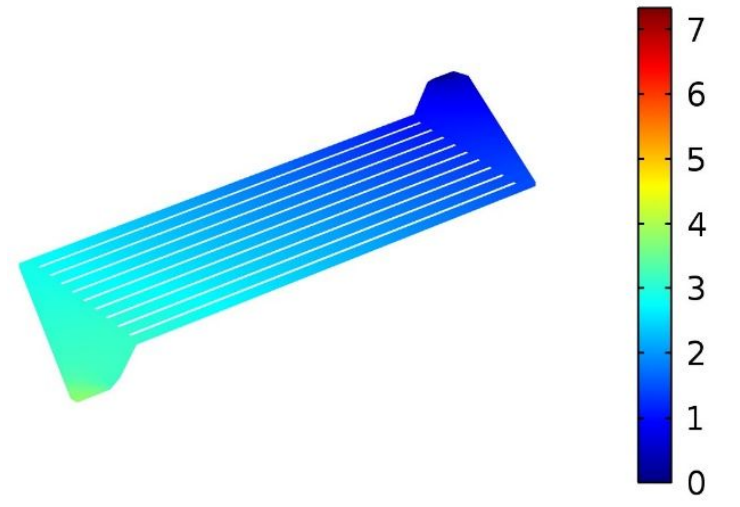

Channel inlet velocity $=40.8 \mathrm{~m} / \mathrm{s}$

(k)

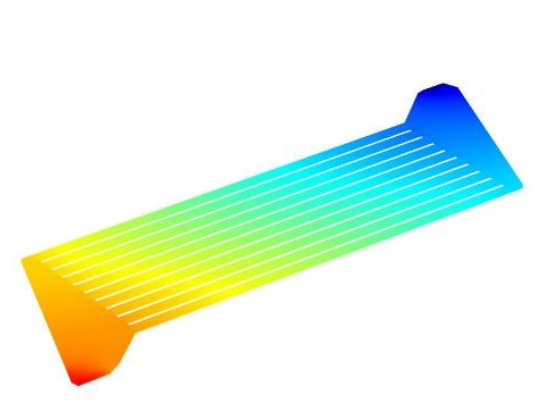

(h)

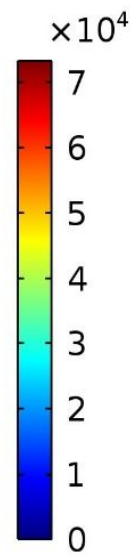

Channel inlet velocity $=38.0 \mathrm{~m} / \mathrm{s}$

(j)

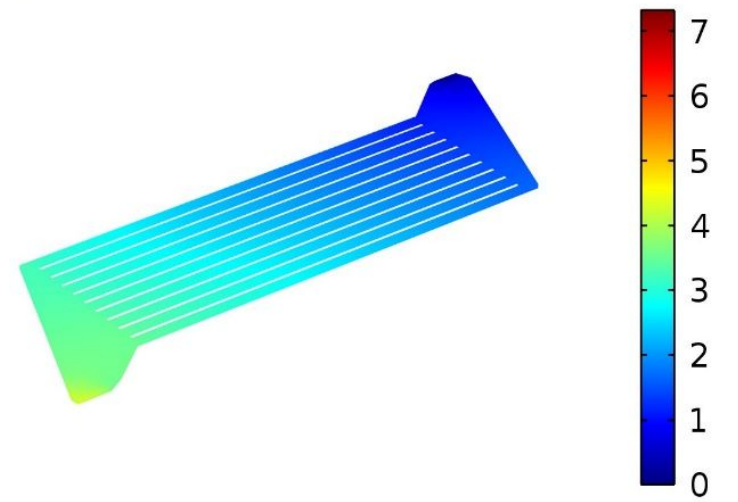

Channel inlet velocity $=43.3 \mathrm{~m} / \mathrm{s}$

(1)

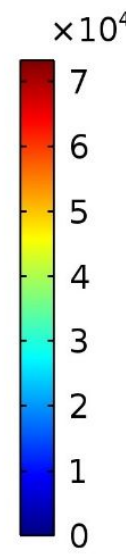

Channel inlet velocity $=57.3 \mathrm{~m} / \mathrm{s}$

Figure S3 $(\mathrm{a}-1)$. The pressure profile in microchannels of catalytic plate reactor at channel inlet velocity from 1.4 to $57.3 \mathrm{~m} / \mathrm{s}$. 
Table S4. Pressure drop across microchannels in catalytic plate reactor.

\begin{tabular}{|c|c|c|c|c|c|c|c|c|c|c|c|}
\hline $\begin{array}{c}\text { Reactor } \\
\text { Inlet } \\
\text { Flow }\end{array}$ & \multicolumn{10}{|c|}{ Pressure drop across microchannels (Pa) } \\
\hline L/min & C-1 & C-2 & C-3 & C-4 & C-5 & C-6 & C-7 & C-8 & C-9 & C-10 & Avg \\
\hline 0.4 & 89 & 88 & 86 & 85 & 83 & 72 & 71 & 79 & 78 & 78 & 81 \\
\hline 1.4 & 651 & 644 & 635 & 625 & 616 & 608 & 600 & 594 & 589 & 586 & 615 \\
\hline 3.2 & 2949 & 2926 & 2886 & 2844 & 2807 & 2412 & 2736 & 2712 & 2693 & 2686 & 2765 \\
\hline 4.0 & 4598 & 4568 & 4503 & 4439 & 4380 & 4321 & 4272 & 4232 & 4206 & 4197 & 4372 \\
\hline 6.4 & 11171 & 11106 & 10953 & 10797 & 10656 & 10512 & 10392 & 10306 & 10250 & 10226 & 10637 \\
\hline 8.7 & 19850 & 19751 & 19481 & 19204 & 18956 & 18695 & 18483 & 17145 & 17154 & 16055 & 18477 \\
\hline 9.8 & 24674 & 24567 & 24234 & 23891 & 23571 & 23242 & 22979 & 22784 & 22684 & 22638 & 23526 \\
\hline 10.3 & 27275 & 27161 & 26793 & 26414 & 26061 & 25697 & 25407 & 25191 & 25082 & 25031 & 26011 \\
\hline 11.1 & 31180 & 31055 & 30635 & 30202 & 29801 & 29383 & 29053 & 28806 & 28684 & 28626 & 29743 \\
\hline 11.8 & 34949 & 34814 & 34344 & 33859 & 33411 & 32942 & 32571 & 32295 & 32161 & 32096 & 33344 \\
\hline 14.7 & 52813 & 52618 & 51909 & 51175 & 50445 & 49662 & 49229 & 48852 & 48631 & 48186 & 50352 \\
\hline 15.7 & 60114 & 59919 & 59112 & 58274 & 57522 & 56704 & 56067 & 55597 & 55386 & 55277 & 57397 \\
\hline
\end{tabular}

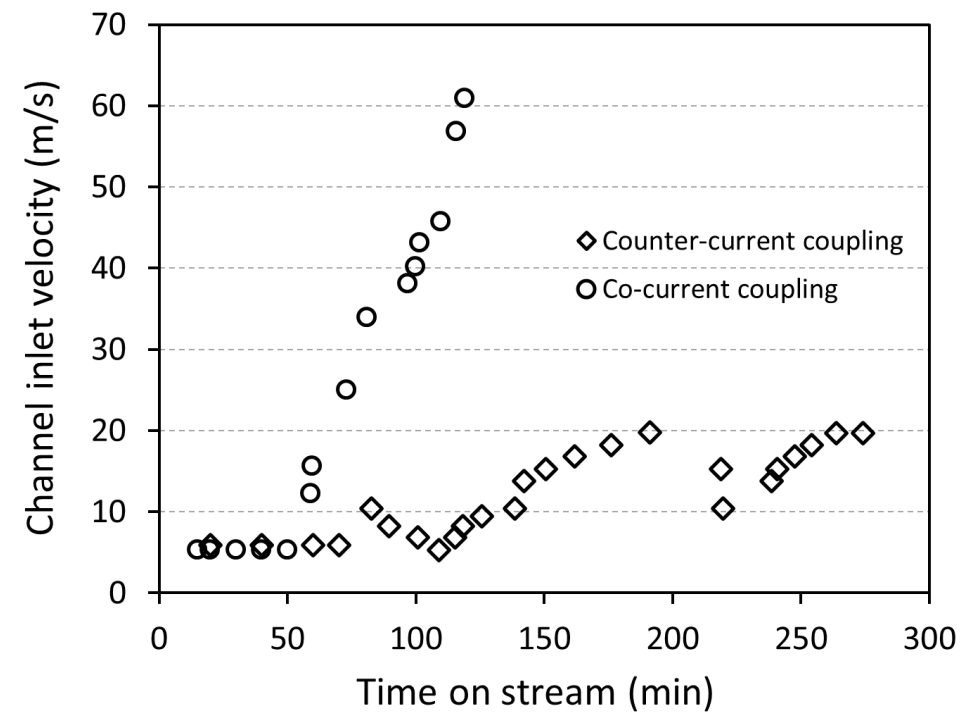

Figure S4: Channel inlet velocity as a function of time on stream (TOS) for coupling of MSR and MC. 


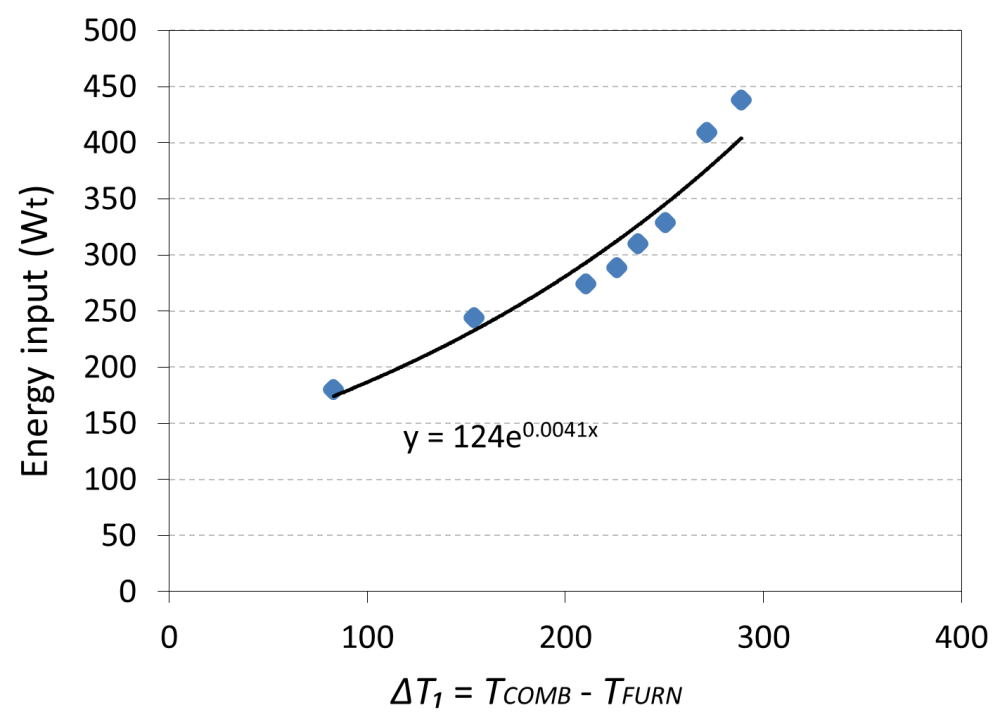

Figure S5: Energy input as a function of $\Delta T_{1}=T_{C O M B}-T_{F U R N}$ for co-current operation.

\section{S2. Second co-current coupling experiment - Furnace heat management}

The feed flow to the reformer consists of methane $(45 \mathrm{~mL} / \mathrm{min})$ and water $(\mathrm{g} / \mathrm{h})$ with $\mathrm{S} / \mathrm{C}$ of 5.0 and WHSV of $172 \mathrm{NL} / \mathrm{h} /$ gcat. In contrast to the other coupling experiments, the combustor's feed flow has a fixed methane flow of $90 \mathrm{~mL} / \mathrm{min}(\lambda=1.5)$, providing a constant energy power of $50 \mathrm{~W}$. The experimental procedure is like the other coupling experiments except after complete methane conversion in the combustor was achieved, the heat required to maintain the reactor temperature was provided from the furnace by turning it $\mathrm{ON}$ and $\mathrm{OFF}$ when $\mathrm{H}_{2}$ production started to decrease.

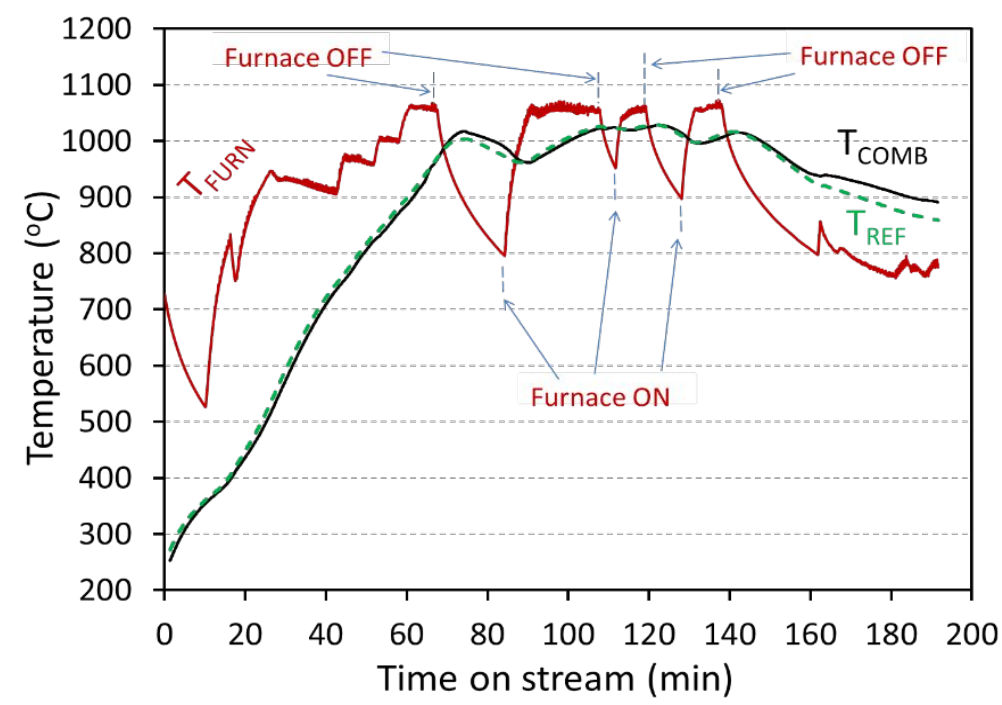

Figure S6: Temperature profile of co-current mode in CPR managed by furnace heat. 


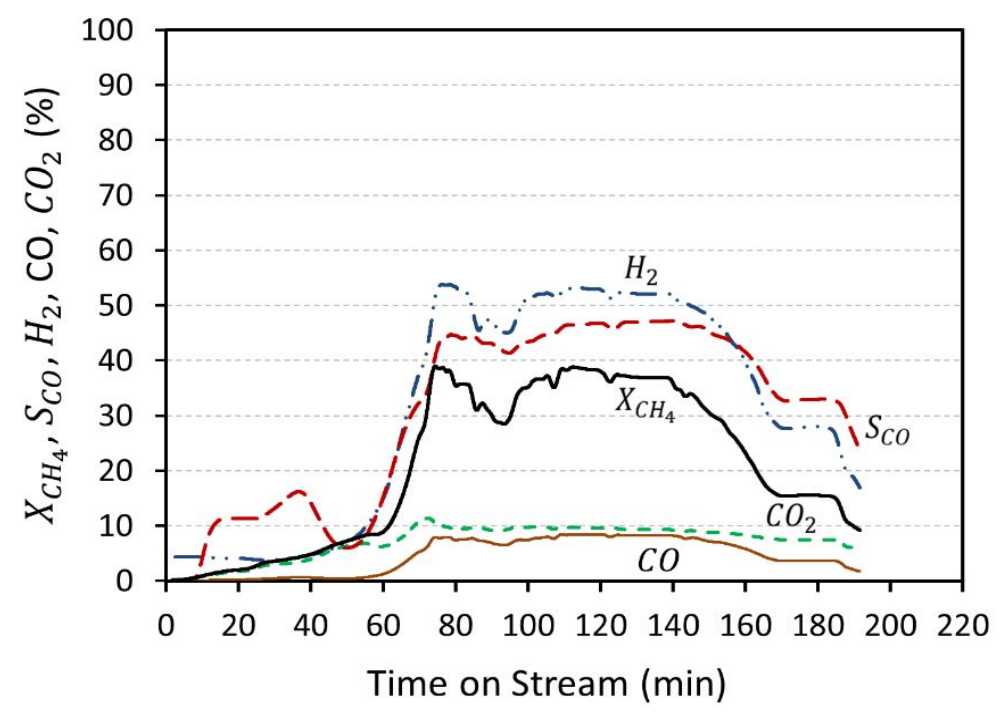

Figure S7: $\mathrm{CH}_{4}$ conversion, $\mathrm{CO}$ selectivity and percent profiles $\left(\mathrm{H}_{2}, \mathrm{CO}\right.$ and $\left.\mathrm{CO}_{2}\right)$ of $\mathrm{MSR}$ for co-current furnace heat managed with a methane flow $(45 \mathrm{~mL} / \mathrm{min})$ and $\mathrm{S} / \mathrm{C}$ of $5.0(172 \mathrm{NL} / \mathrm{h} /$ gcat $)$.

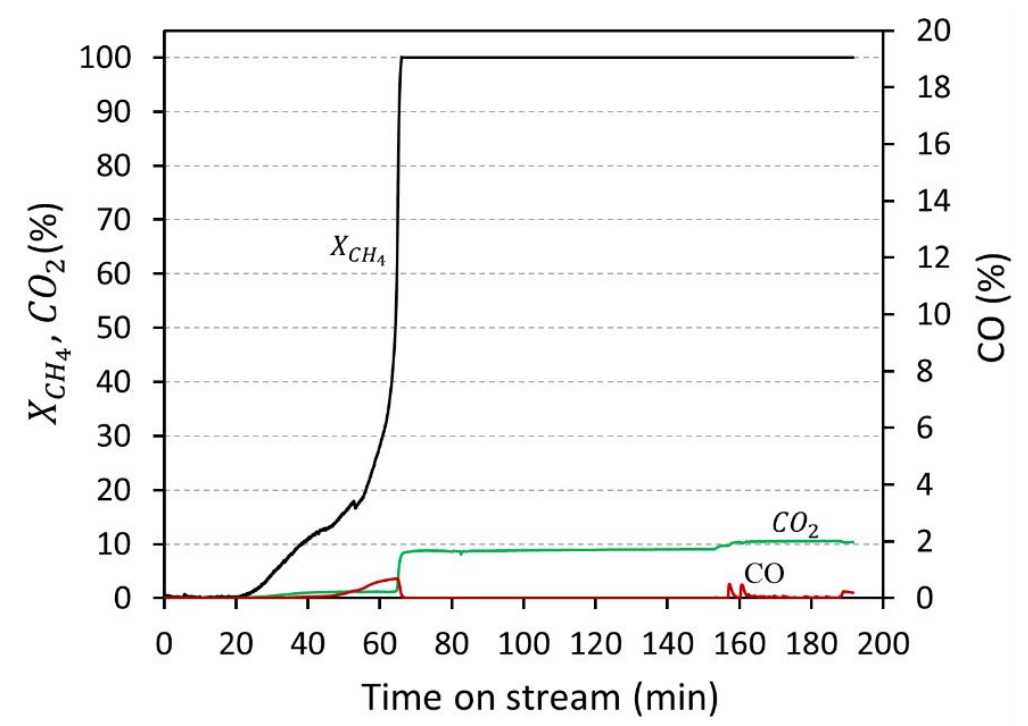

Figure S8: Methane conversion and concentration profiles in the combustion side in co-current operation as a function of time on stream (furnace heat managed). 


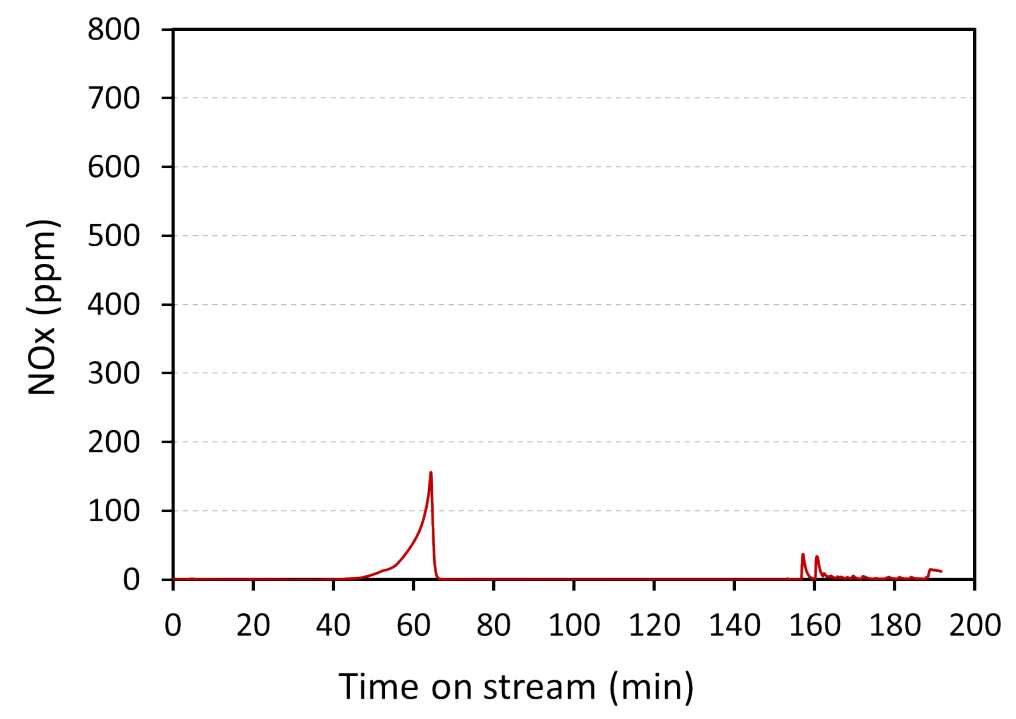

Figure S9: The concentration of $\mathrm{N}_{2} \mathrm{O}(\mathrm{ppm})$ in co-current mode in CPR (furnace heat managed).

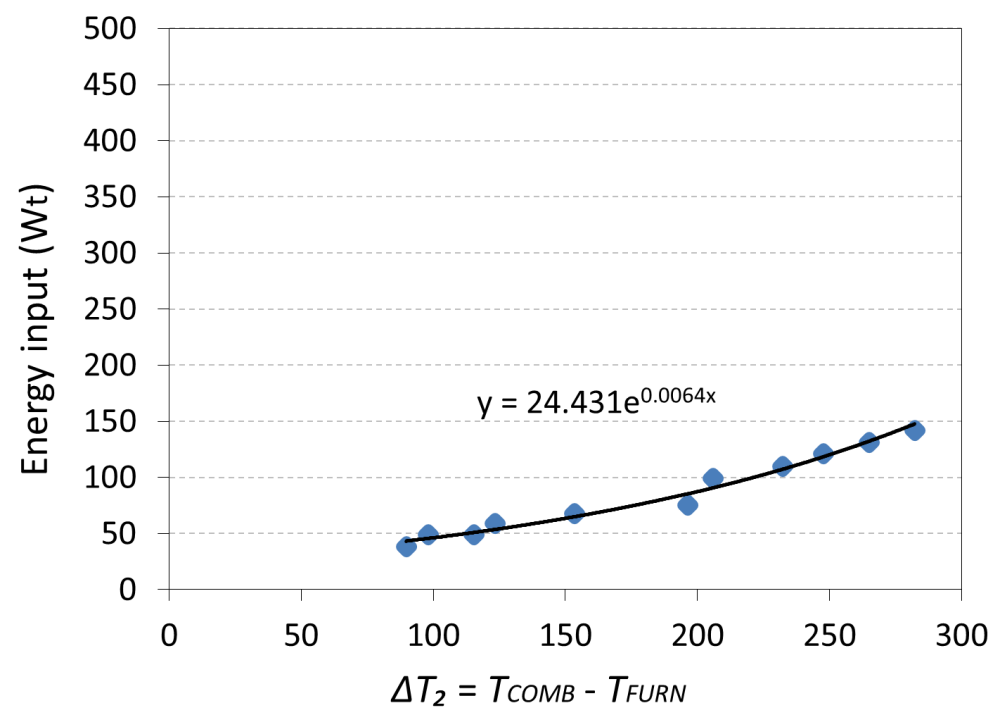

Figure S10: Energy input as a function of $\Delta T_{2}=T_{\text {COMB }}-T_{F U R N}$ for counter-current operation. 


\section{S3. Second counter-current coupling experiment}

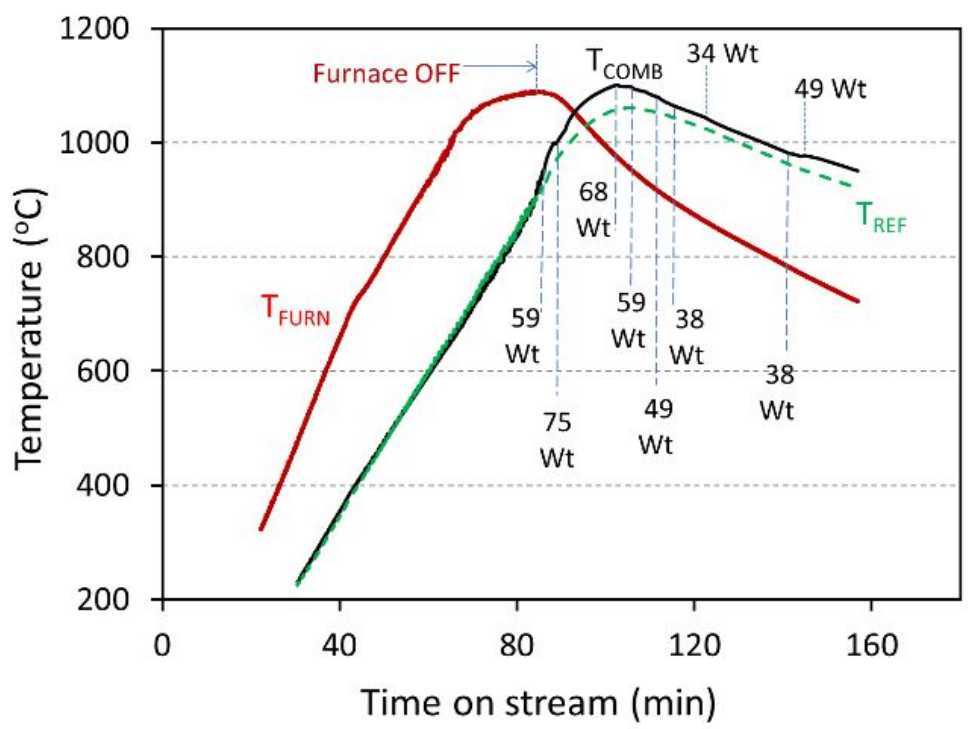

Figure S11. The temperature profile for second counter current coupling operation.

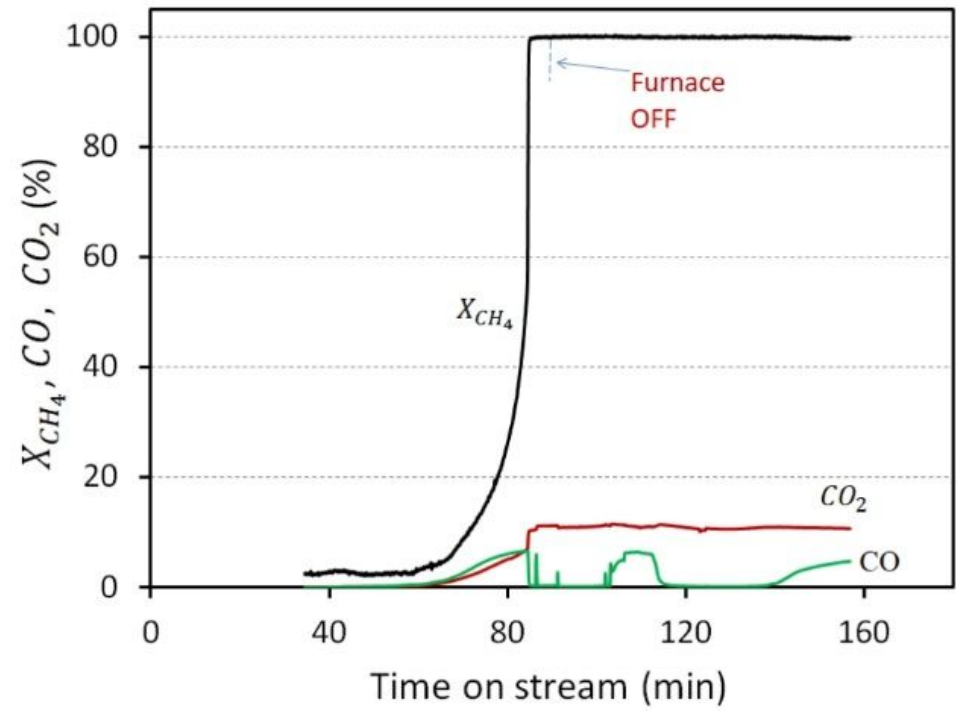

Figure S12. $\mathrm{CH}_{4}$ conversion and concentration profiles of on combustion side for counter-current operation as a function of time on stream. 


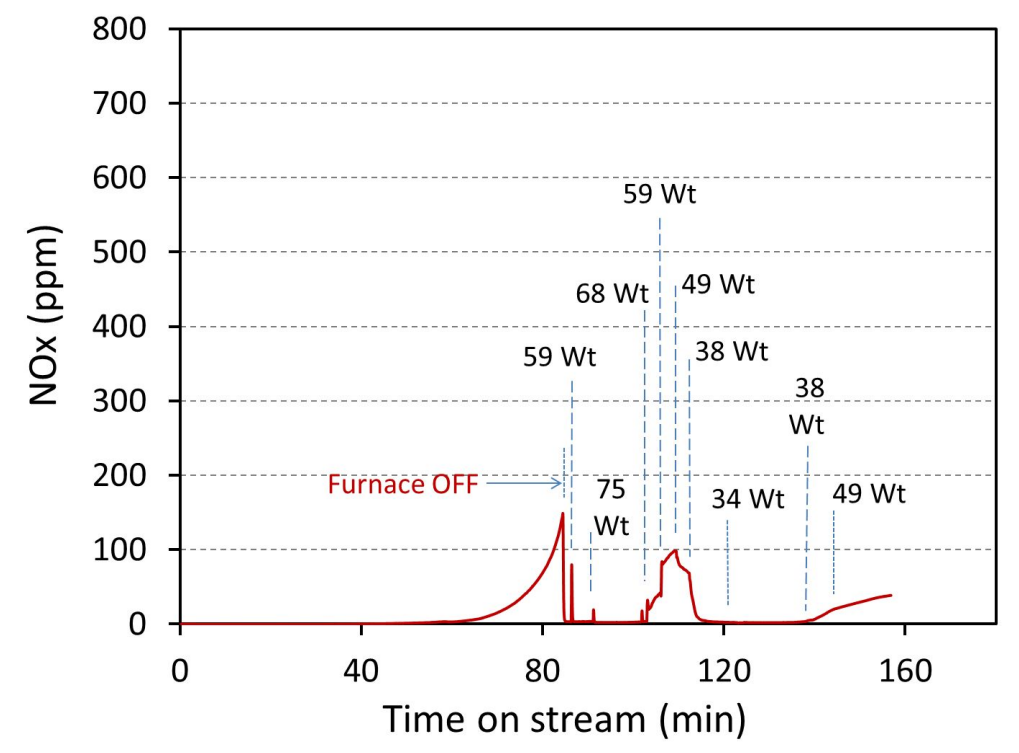

Figure $\mathrm{S} 13$. The concentration of $\mathrm{N}_{2} \mathrm{O}(\mathrm{ppm})$ for counter-current mode in catalytic plate reactor.

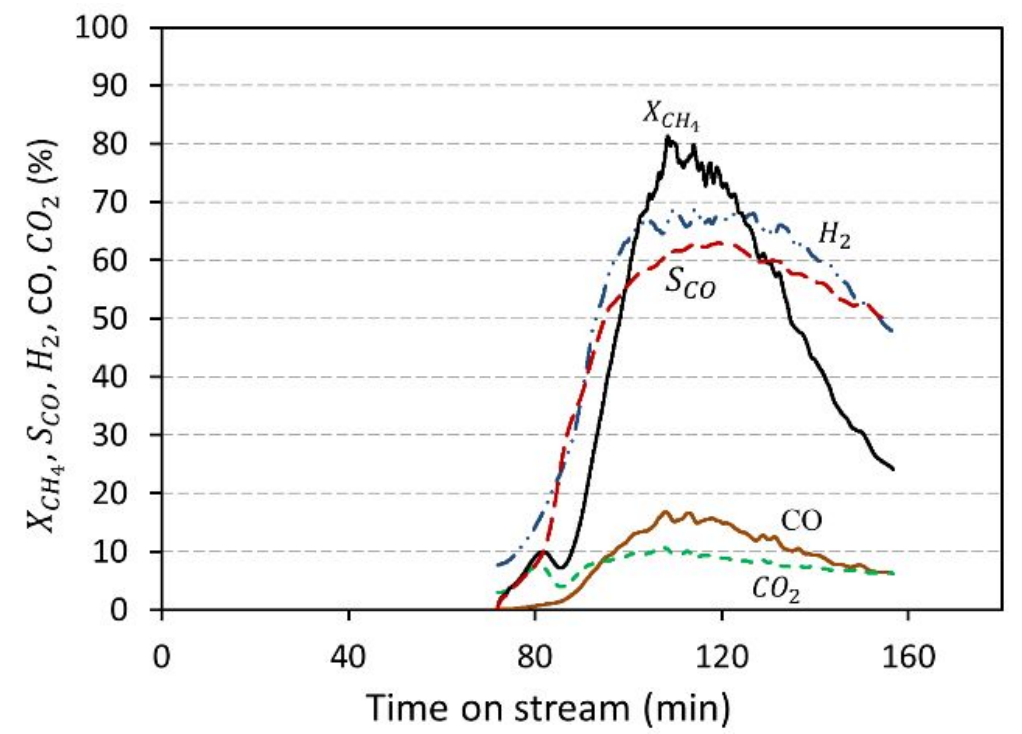

Figure $\mathrm{S} 14 . \mathrm{CH}_{4}$ conversion, $\mathrm{CO}$ selectivity and concentration profiles $\left(\mathrm{CO}, \mathrm{CO}_{2}\right.$ and $\left.\mathrm{H}_{2}\right)$ of methane steam reforming for counter-current flow mode with methane flow rate of $45 \mathrm{~mL} / \mathrm{min}$ and $\mathrm{S} / \mathrm{C}$ molar ratio of 4.0 . 